\title{
Maxillary Occlusion Rehabilitation of Multiple Anterior Teeth Agenesis Followed by Tooth Wear: A Case Report
}

\author{
Muhammad Dimas Aditya Ari, DDS, MSc, ${ }^{1}$ Abil Kurdi, DDS, ${ }^{1}$ \\ Yonatan Christian Suisan, DDS ${ }^{2}$ and Harry Laksono, DDS MSc ${ }^{1}$ \\ ${ }^{1}$ Department of Prosthodontics, Faculty of Dental Medicine, University of Airlangga, Surabaya, Indonesia \\ ${ }^{2}$ Resident of Prosthodontics, Faculty of Dental Medicine, University of Airlangga, Surabaya, Indonesia
}

\begin{abstract}
A 19-year-old female patient came with the chief complaint that the upper anterior teeth looked small and with many spaces. The patient claimed that the tooth had never been replaced since birth. The patient wanted to improve the aesthetics of the tooth. Treatment, in this case, was made using a fixed prosthetic to aesthetically rehabilitate through prosthodontic treatment in the anterior teeth agenesis followed by attrition. It can be concluded that fixed prosthodontic treatment using a splint bridge is one treatment option that can be used in multiple anterior teeth agenesis followed by tooth wear. An appropriate material selection increases the aesthetics and functional aspects of the final treatment.
\end{abstract}

Key Words: fixed partial denture, anodontia, dental occlusion, human, health

\section{INTRODUCTION}

Nowadays, aesthetic dentistry is growing and starting to be in great demand in line with technological and scientific developments in the medical field, especially dentistry. With this development, a shift in demand and the community's need for dental care also transpired. Aesthetics is also one of the crucial considerations in treatment that has been focused on minimizing pain. So, in prosthetic dentistry and focusing on dental, medical rehabilitation on the restoration of stomatognathic function holistically, improving aesthetic appearance is also included in the current needs. ${ }^{1}$

Facial aesthetics are closely related to the harmonious arrangement of teeth. Several components need to be considered, i.e., the health and proportion of teeth and gingival tissue, the smile arc, the composition and color of the teeth. The maxilla's left and right central incisors are the primary keys to determining the anterior region's aesthetics. ${ }^{1,2}$ One of the anomalies that often occur in tooth development that can interfere with aesthetic function is tooth agenesis. ${ }^{3}$

Tooth agenesis can occur in primary and permanent teeth

Paper presented in the Temu IImiah Nasional dan Internasional ke-8 (TIMNASS 8) - Faculty of Dental Medicine, Universitas Airlangga, Surabaya, Indonesia.

Corresponding author: Muhammad Dimas Aditya Ari, DDS MSc Department of Prosthodontics

Faculty of Dental Medicine

University of Airlangga

Surabaya, Indonesia

Email: dimasadityadrg@fkg.unair.ac.id and can be diagnosed through routine clinical examination and radiography. The reported incidence of dental agenesis is $3 \%-10 \%$, with an increased incidence in women..$^{4-6}$ The most common incident occurs in the third molars, followed by the premolars, then the lateral maxillary incisors. ${ }^{5,7}$ Tooth agenesis in the anterior region creates an aesthetic problem. ${ }^{8}$ Aesthetically, tooth agenesis gives an unfavorable appearance that decreases a patient's self-confidence in persons with 
tooth agenesis. There are several approaches to managing tooth agenesis cases. If tooth agenesis occurs in 1 or 2 teeth, the treatment can be space closing through orthodontic treatment. ${ }^{9}$ In some cases, a selection of treatments is in demand, and using a prosthetic approach is increasing. ${ }^{10,11}$ In tooth agenesis, deciduous teeth in the arch may be used in the stomatognathic function. Anatomically, the structure of primary teeth that are more vulnerable than permanent teeth makes primary teeth in tooth agenesis cases often experience tooth wear and craniofacial growth. ${ }^{12,13}$ This mainly occurs in tooth agenesis cases that lasted a long time without proper treatment. Tooth wear in the management of prosthodontic cases remains a significant challenge.

This case report discusses the management of multiple maxillary tooth agenesis followed by attrition through a prosthodontic approach.

\section{CASE REPORT}

A 19-year-old female patient came to the Prosthodontics Clinic at Universitas Airlangga Dental Hospital with the chief complaint that the upper anterior teeth looked small and many spaces. The patient claimed that the tooth had never been replaced since birth. According to the mother, there was no history of syndromic or systemic disease. There was no family history with the same condition. The patient wanted to improve the aesthetics of the tooth. The patient had never used a denture before. Clinical examination showed that there were multiple agenesis of teeth $15,14,12,11,21$, $22,25,33,32,31,41,42,43,45$ and persistence of teeth 52 , $51,61,62,63,73,72,71,81,82,83,85$. These clinical findings are confirmed by panoramic radiography. Tooth wear was shown in teeth 51,52, and 61 and was diagnosed as reversible pulpitis caused by non-caries lesion followed by excessive gum. The permanent teeth of this patient were 13, 16, 17, $23,25,26,33,35,36,43$ and 46 . The overjet and overbite were $0 \mathrm{~mm}$. Based on clinical examination of the occlusion, there was no decrease in vertical dimensions of occlusion. The maximum unassisted and pain-free mouth opening was 34 $\mathrm{mm}$, lateral movement to the right and left was $6 \mathrm{~mm}$, and protrusive movement was $7 \mathrm{~mm}$. All of the measurements ranged in a normal movement. There were no deviation and/or deflection of the mandible, clicking and pain in the temporomandibular joint, tenderness in the mouth opening and closing muscle. The pre-operative clinical view of the patient can be seen in Figure 1.

\section{CASE MANAGEMENT}

Treatment options were explained to the patient. She agreed to a fixed prosthetic approach. The patient signed informed consent before treatment started. The preliminary impression was then taken using a stock tray and an irreversible hydrocolloid material. The maxillary impression was cast using type IV gypsum to prevent a fracture of the study model's remaining mandibular tooth elements; the

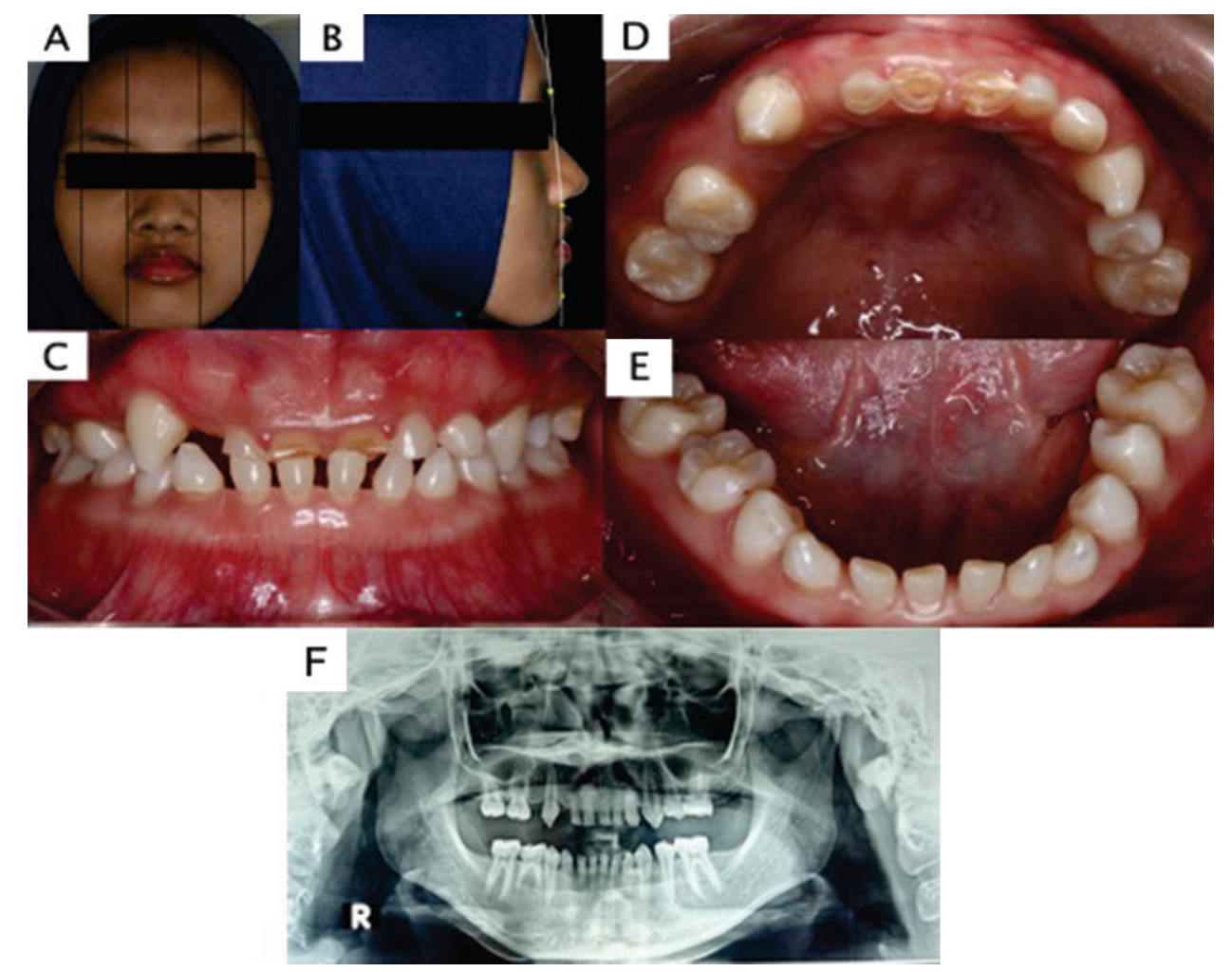

Figure 1. Clinical (A-E) and radiological panoramic (F) view of the patient. 
mandibular impression was then cast using acrylic. The face bow transfer was performed using Universal Transfer System (UTS) 3D with bite fork (Stratos 300, Ivoclar Vivadent). The enhancement of vertical dimension occlusion of about 1 $\mathrm{mm}$ was performed to make an interincisal space according to clinical vertical dimension occlusion measurement with 3 $\mathrm{mm}$ of free-way space. A $1 \mathrm{~mm}$ occlusal stop using acrylic was placed in the anterior region, and a bite registration was done. The maxillary study model was mounted in the semi-adjustable articulator (Stratos 300, Ivoclar Vivadent) using UTS 3D guidance. Figure 2 shows the process of the enhancement of the vertical dimension of occlusion mounted to the articulator. The mandibular study model was mounted to the articulator with the bite registration using the anterior stop. Study model analysis and occlusion were performed, and the wax-up was made for the patient.

The waxed-up model was discussed on the patient's second visit. The patient agreed on the proportion and shape of the teeth. Tooth preparation of the posterior teeth was then done in 13,16, 23, 24, and 26. After tooth preparation, the posterior final prosthesis's final impression was performed using the putty-wash technique with putty and light body. The 4-units fixed-fixed bridge with porcelain fused to metal was made for teeth $13,14,16$, and 17, and a 3 -unit splint crown with porcelain fused to metal material for teeth 23,24 , and 26 was made in the laboratory. The 4-units fixed-fixed bridge and three units splint crown for the posterior maxillary teeth were then cemented using temporary luting cement (Freegenol Temporary Pack, GC). The patient underwent this condition for a week to adapt to the new occlusion.

A week after the posterior fixed prosthesis was temporarily cemented, the patient was asked for any complaints during the adaptation period. After no objections were received, the posterior teeth were cemented using luting
GIC. The anterior teeth preparation was then performed with the mock-up-driven technique (Figure 3). After the teeth preparation was done on $52,51,61,62$, and 63 , the anterior prosthesis's final impression was performed using the putty-wash technique with putty and light body. The six units fixed-fixed splint bridge with composite materials for teeth $53,52,51,61,62$, and 63 was made in the laboratory. The patient used a temporary splint bridge until the laboratory-fabricated permanent six units fixed-fixed splint bridge. The six units fixed-fixed splint bridge using composite materials were then cemented with temporary luting cement (Freegenol Temporary Pack, GC). The patient underwent this condition for a week to adapt to the new occlusion. With no additional complaints, the final cementation for anterior teeth was performed using dual-cure resin luting material (Figure 4) on the next visit.

\section{DISCUSSION}

Fixed prosthodontics involves the replacement and restoration of the teeth with artificial material that cannot be removed. This prosthesis was used to restore function, enhance aesthetics, and comfort patients with tooth loss or development anomalies like agenesis. Fixed prosthodontics offers better satisfaction by restoring stomatognathic function and poor clinical appearance to a comfortable and healthy occlusion that can last a long time by considering the aesthetic aspects. ${ }^{1}$

As a space for restoration in cases with overjet and overbite of $0 \mathrm{~mm}$, the vertical dimension of occlusion (VDO) was needed. Several studies have written that increasing VDO is one method that can facilitate restorative treatment and a way to get space in dental aesthetic treatment. ${ }^{14,15} \mathrm{~A}$ technique that can be used to improve VDO is free-way space measurement. A free-way space of $2 \mathrm{~mm}$ has been

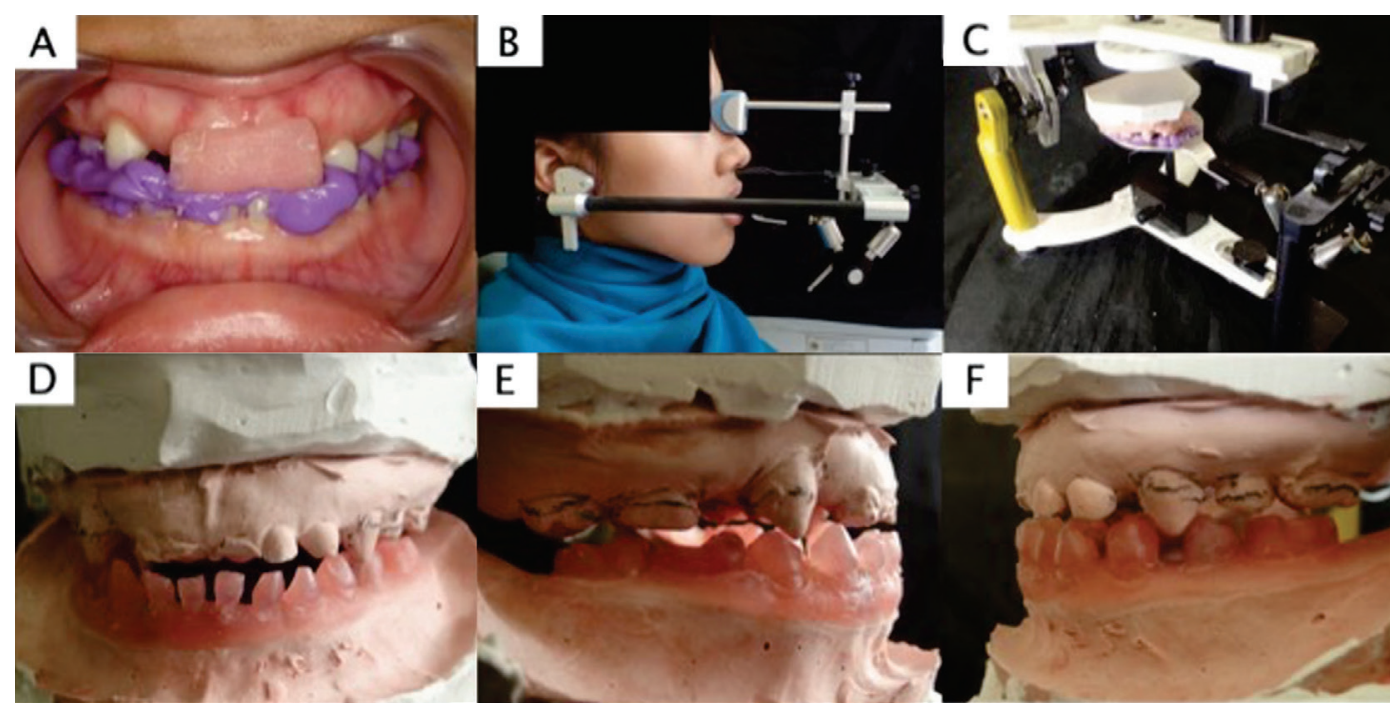

Figure 2. The enhancement of vertical dimension of occlusion (A), face-bow transfer (B), and mounting to the semi-adjustable articulator (C-F). 


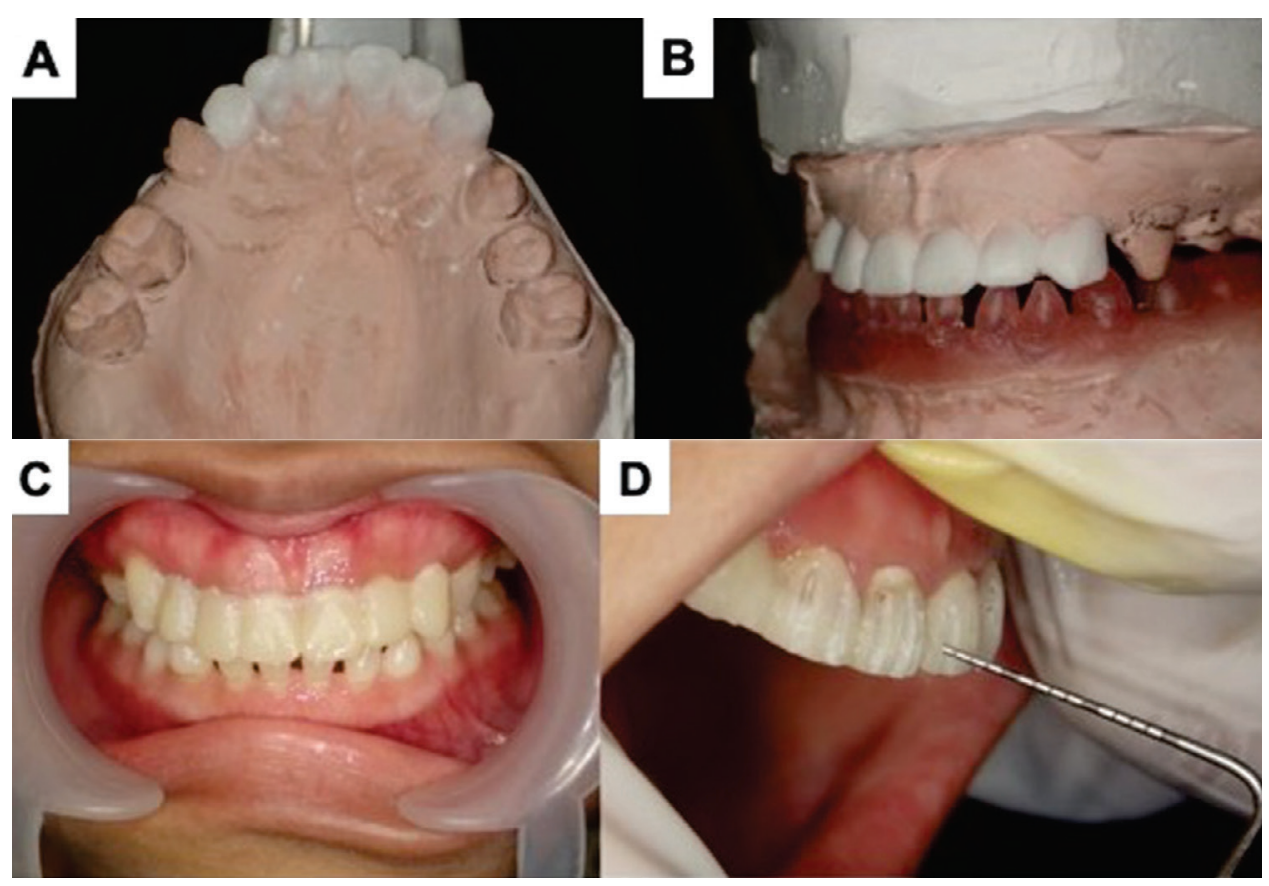

Figure 3. The wax-up model (A-B), mock-up to the patient (C), and mock-up-driven technique preparation (D).

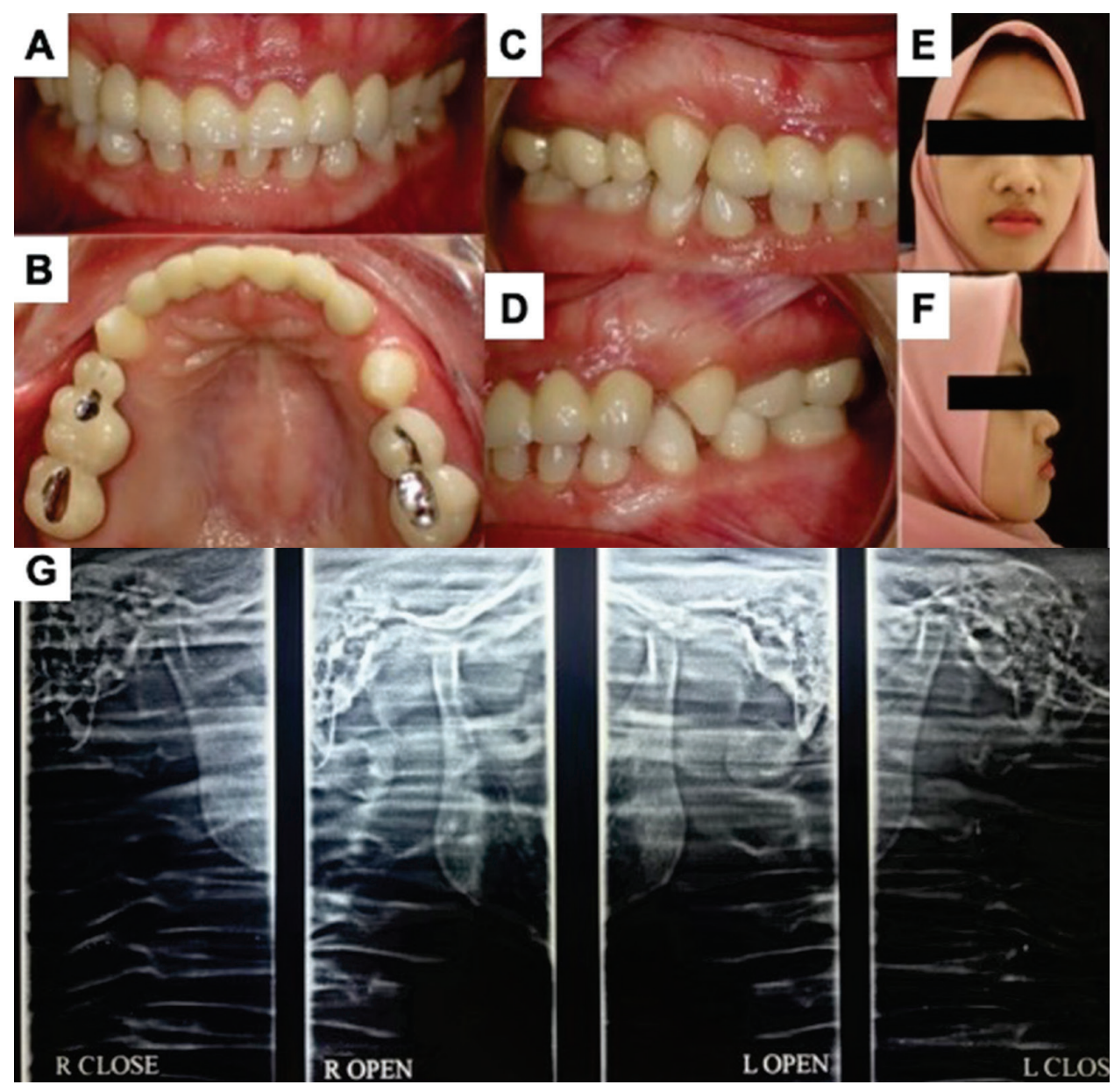

Figure 4. Final prosthesis (A-D), clinical profile of patient (E-F), and evaluation from TMJ (G). 
Maxillary Occlusion Rehabilitation

suggested as a physiological space, and therefore, a freeway space over $2 \mathrm{~mm}$ indicates that VDO can be safely increased. ${ }^{16}$ In these patients with a free-way space of $3 \mathrm{~mm}$, an increase in VDO can be done by $1 \mathrm{~mm}$. A related study showed that an increase in VDO could be done up to $5 \mathrm{~mm}$. Permanent prosthetic treatment with a maximum increase in VDO of $5 \mathrm{~mm}$ is safe and predictable without any adverse consequences. ${ }^{16}$ In tooth-supported prosthetics, a study showed that a reasonable and predictable adaptation phase occurs at 1-2 weeks post elevated VDO. ${ }^{17}$

In this case, a semi-adjustable articulator was used alongside the use of face-bow transfers. The use of semiadjustable articulators can record facial areas through facebow transfers and interocclusal, adjusted according to the individual. This instrument has condylar guidance that can be adjusted individually, both horizontally and vertically. ${ }^{18}$ In general, the use of semi-adjustable articulators provides good treatment results, especially in cases that require prosthetic accuracy, such as VDO elevation. The advantage of using a semi-adjustable articulator accompanied by facebow transfer is that it allows the transfer of retruded path of closure, allows condylar transfer angle to the position of the mandibular protrusion and moves laterally, allows lateral displacement approaching the original state of the patient, and the incisal guidance table can be arranged. ${ }^{19}$

Persistent primary teeth are retained because of good root conditions based on clinical and radiographic examinations. There was no mobility, and the roots were still well embedded in the alveolar bone. Consideration of the patient's age is also one factor in deciduous teeth whose persistence is still maintained and planned to be used as an abutment in fixed prosthodontics.

In a related study, the crown-root ratio (CRR) is required to obtain fixed prosthodontic treatment. CRR is measured in relation to the alveolar bone support, which is quantified by dividing the crown portion's length by the length of the root portion. The greater proportion of roots embedded in the alveolar bone, the more ideal the tooth is used as an abutment in fixed prosthodontics. ${ }^{20}$

\section{CONCLUSION}

From this case, it can be concluded that fixed prosthodontic treatment using a splint bridge is one treatment option that can be used in multiple anterior teeth agenesis followed by tooth wear. An appropriate material selection increases the esthetic and functional aspects of the final treatment.

\section{Statement of Authorship}

All authors participated in the data collection and analysis and approved the final version submitted.

\section{Author Disclosure}

All authors declared no conflicts of interest.

\section{Funding Source}

No funding support.

\section{REFERENCES}

1. Rosentiel SF, Land MF, Fujimoto J. Contemporary Fixed Prosthodontics. 2nd ed. St. Louis, Missouri: Elsevier Academic Press; 2015.

2. Ward DH. A study of dentists' preferred maxillary anterior tooth width proportions: comparing the recurring esthetic dental proportion to other mathematical and naturally occurring proportions. Journal of Esthetic and Restorative Dentistry. 2007;19(6):324-39.

3. Ritwik P, Patterson KK. Diagnosis of tooth agenesis in childhood and risk for neoplasms in adulthood. Ochsner Journal. 2018;18(4): $345-50$.

4. Ye X, Attaie AB. Genetic basis of nonsyndromic and syndromic tooth agenesis. Journal of Pediatric Genetics. 2016;5(04):198-208.

5. Jonsson L, Magnusson TE, Thordarson A, Jonsson T, Geller F, Feenstra $\mathrm{B}$, et al. Rare and common variants conferring risk of tooth agenesis. Journal of Dental Research. 2018;97(5):515-22.

6. Iavazzo C, Papakiritsis M, Gkegkes ID. Hypodontia and ovarian cancer: a systematic review. Journal of the Turkish German Gynecological Association. 2016;17(1):41.

7. De Coster PJ, Marks LA, Martens LC, Huysseune A. Dental agenesis: genetic and clinical perspectives. Journal of Oral Pathology \& Medicine. 2009;38(1):1-7.

8. Almeida RR, Morandini AC, Almeida-Pedrin RR, Almeida MR, Castro RC, Insabralde NM. A multidisciplinary treatment of congenitally missing maxillary lateral incisors: a 14-year follow-up case report. Journal of Applied Oral Science. 2014;22(5):465-71.

9. Bilgin N, Kaya B. Etiology and treatment alternatives in tooth agenesis: a comprehensive review. Stomatological Disease and Science. 2018;2(9):1-11.

10. Zarone F, Sorrentino R, Vaccaro F, Russo S. Prosthetic treatment of maxillary lateral incisor agenesis with osseointegrated implants: a 2439-month prospective clinical study. Clinical Oral Implants Research. 2006;17(1):94-101.

11. Goshima K, Lexner MO, Thomsen CE, Miura H, Gotfredsen K, Bakke M. Functional aspects of treatment with implant-supported single crowns: a quality control study in subjects with tooth agenesis. Clinical Oral Implants Research. 2010;21(1):108-14.

12. Isidro S, Ono Y, Takagi Y. Craniofacial growth changes and dental attrition in the primary dentition. Pediatric Dental Journal. 2012;22(1):43-9.

13. Warren JJ, Yonezu T, Bishara SE. Tooth wear patterns in the deciduous dentition. American Journal of Orthodontics and Dentofacial Orthopedics. 2002;122(6):614-8.

14. Keough B. Occlusion-based treatment planning for complex dental restorations: Part 1. International Journal of Periodontics \& Restorative Dentistry. 2003;23(3):237-47.

15. Abduo J, Lyons K. Clinical considerations for increasing occlusal vertical dimension: a review. Australian Dental Journal. 2012;57(1): $2-10$.

16. Abduo J. Safety of increasing vertical dimension of occlusion: A systematic review. Quintessence international. 2012;43(5):369-80.

17. Ormianer Z, Palty A. Altered vertical dimension of occlusion: a comparative retrospective pilot study of tooth-and implant-supported restorations. International Journal of Oral \& Maxillofacial Implants. 2009;24(3):497-501.

18. Klineberg I, Eckert SE, Zarb G. Functional Occlusion In Restorative Dentistry and Prosthodontics. St Louis Missouri: Elsevier Inc.; 2016.

19. Thomson H. Occlusion. 1st ed. Great Britain: John Wright and Sons Limited.; 2001.

20. Tada S, Allen PF, Ikebe K, Zheng H, Shintani A, Maeda Y. The impact of the crown-root ratio on survival of abutment teeth for dentures. Journal of Dental Research. 2015;94(9):220-5. 